\title{
Correction to: Cryptosporidium Spp. Contamination in Perna perna Mussels Destined for Human Consumption in Southeastern Rio de Janeiro, Brazil
}

\author{
A. L. Santos ${ }^{1}$ - L. T. F. de Oliveira ${ }^{2} \cdot$ A. L. A. Souza ${ }^{1} \cdot$ R. A. Hauser-Davis ${ }^{3} \cdot$ S. G. De Simone ${ }^{4}$ \\ Published online: 14 February 2018 \\ (c) Springer Science+Business Media, LLC, part of Springer Nature 2018
}

\section{Correction to: \\ Bulletin of Environmental Contamination and Toxicology https://doi.org/10.1007/s00128-017-2223-2}

The original version of this article unfortunately contained a mistake. One of the affiliations was incorrect. The corrected affiliation for the author S.G. De Simone is Centro de Desenvolvimento Tecnológico em Saúde (CDTS) - FIOCRUZ, Rio de Janeiro, RJ CEP 21040-361 Brazil.

The original article can be found online at https://doi.org/10.1007/ s00128-017-2223-2.

A. L. Santos

antonia@ioc.fiocruz.br

1 Laboratório de Bioquímica Experimental e Computacional de Fármacos - IOC FIOCRUZ, Rio de Janeiro, RJ CEP 21040-360, Brazil

2 Escola Politécnica de Saúde Joaquim Venâncio - EPSJV PROVOC - FIOCRUZ, Rio de Janeiro, RJ 21045-900, Brazil

3 Centro de Estudos da Saúde do Trabalhador e Ecologia Humana, Escola Nacional de Saúde Pública, Fundação Oswaldo Cruz, Rio de Janeiro, RJ CEP 21041210, Brazil

4 Centro de Desenvolvimento Tecnológico em Saúde - FIOCRUZ, Rio de Janeiro, RJ CEP 21040-361, Brazil 